\title{
ISOTERMAS E CALOR ISOSTÉRICO DE SORÇÃO DO FEIJÃO ${ }^{1}$
}

\author{
Osvaldo RESENDE ${ }^{2, *}$, Paulo Cesar CORRÊA ${ }^{2}$, André Luis Duarte GONELI², Deise Menezes RIBEIRO²
}

\begin{abstract}
RESUMO
O teor de água de equilíbrio dos grãos de feijão foi determinado pelo método dinâmico-gravimétrico para temperaturas entre 25 e $55{ }^{\circ} \mathrm{C}$ e atividades de água para cada temperatura, entre 0,20 e 0,75 . Observou-se que o teor de água decresce com o aumento da temperatura para uma dada atividade de água à semelhança dos produtos higroscópicos. Aos dados experimentais foram ajustados diversos modelos matemáticos disponíveis na literatura (Brunauer-Emmett-Teller, Chung Pfost, Copace, Guggenhein-Anderson-de Boer, Halsey Modificado, Henderson, Henderson Modificado, Oswin, Sabbah e Sigma Copace). Segundo os parâmetros estatísticos de análise, o modelo de Halsey Modificado foi o que descreveu melhor a higroscopicidade do feijão. A partir dos resultados obtidos calculou-se o calor isostérico para cada teor de água de equilíbrio. Observou-se que o calor isostérico aumenta com a diminuição do conteúdo de água do produto, indicando que a redução do teor de água aumenta a energia necessária para a remoção de água. Os valores de calor isostérico para os grãos de feijão na faixa de umidade de 10,10 a 21,71 (\% b.s.), variam de 3961 a $2718 \mathrm{~kJ}^{\mathrm{kg}}{ }^{-1}$.

Palavras-chave: feijão, higroscopicidade, modelagem matemática, dessorção.
\end{abstract}

\section{SUMMARY}

ISOTHERMS AND ISOSTERIC HEATS OF SORPTIONS OF THE EDIBLE BEAN. The equilibrium moisture content of the edible bean was determined using the dynamic-gravimetric method for temperatures between 25 and $55{ }^{\circ} \mathrm{C}$ and water activity between 0.20 and 0.75. It was observed that the moisture content decreases when the temperature for a given water activity is increased. Experimental data were adjusted to mathematical models mentioned in the specific literature (Brunauer-Emmett-Teller, Chung Pfost, Copace, Guggenhein-Anderson-de Boer, Modified Halsey, Henderson, Modified Henderson, Oswin, Sabbah and Sigma Copace). According to the results obtained and statistical parameters, the Modified Halsey model was the most effective in representing the hygroscopicity of the edible bean. From the obtained results, the desorption isosteric heats for each equilibrium moisture content was calculated. It was observed that the desorption isosteric heat increases when the moisture content decreases indicating that the reduction in the moisture content increases the energy needed to remove water. The values of isosteric heats for the edible bean in the moisture content are between 10.10 to 21.71 (\% b.s.) and they vary from 3961 to $2718 \mathrm{~kJ} \mathrm{~kg}^{-1}$.

Keywords: edible bean, higroscopicity, mathematical modeling, desorption.

\section{1 - INTRODUÇÃO}

Durante o armazenamento do feijão podem ocorrer mudanças físicas, químicas e microbiológicas que, dependendo da interação entre estes fatores e o ambiente, podem ocasionar perdas na sua qualidade. Assim, faz-se necessário o conhecimento das relações existentes entre o produto, a temperatura e a umidade relativa do ar, objetivando iniciativas e estudos com a finalidade de amenizar estas possíveis alterações.

Todos os produtos agrícolas têm a capacidade de ceder ou absorver água do ambiente, convergindo, constantemente, para uma relação de equilíbrio entre o seu teor de água e as condições do ar ambiente.

O teor de água de equilíbrio é alcançado quando a pressão parcial de vapor de água no produto iguala-se a do ar que o envolve. A relação entre o teor de água de um determinado produto e a umidade relativa de equilíbrio para uma temperatura específica pode ser expressa por meio de

\footnotetext{
${ }^{1}$ Recebido para publicação em 9/9/2005. Aceito para publicação em 6/7/2006 (001608)

${ }^{2}$ Departamento de Engenharia Agrícola (CENTREINAR/UFV),

Av. P. H. Rolfs s/n, campus (UFV), CEP 36570-000, Viçosa (MG),

E-mails: osvresende@yahoo.com.br,copace@ufv.br,

andregoneli@yahoo.com.br,deise_eng@yahoo.com.br

* A quem a correspondência deve ser enviada
}

equações matemáticas denominadas isotermas ou curvas de equilíbrio higroscópico.

O comportamento higroscópico de diversos produtos agrícolas tem sido estudado por vários pesquisadores, que descrevem modelos diferenciados para expressar o teor de água de equilíbrio em função da temperatura e umidade relativa do ar. Entretanto, para o estabelecimento de isotermas que representam essa relação de equilíbrio são utilizados modelos matemáticos empíricos, uma vez que nenhum modelo teórico desenvolvido tem sido capaz de predizer com precisão o teor de água de equilíbrio para uma ampla faixa de temperatura e umidade relativa do ar.

Atualmente, na literatura, existem mais de 200 equações propostas para representar o fenômeno de equilíbrio higroscópico dos produtos agrícolas. Estes modelos diferem na sua base teórica ou empírica e na quantidade de parâmetros envolvidos [10].

Segundo WANG \& BRENNAN [13], para remover a umidade associada a um material higroscópico, a energia necessária é maior do que a utilizada para vaporizar igual quantidade de água livre, nas mesmas condições de pressão e temperatura. Esse adicional de energia, em virtude das forças de ligação entre a água e a superfície da substância adsorvente, é denominado calor isostérico de dessorção nos processos de secagem, constituindo-se em um bom parâme- 
tro para se estimar a quantidade mínima de calor requerida para remover uma quantidade de água do produto.

De acordo com MULET et al. [9], o teor de água em que o calor isostérico de sorção é quase idêntico ao calor latente de vaporização da água livre, é geralmente considerado como um indicador do conteúdo de água livre no produto. Segundo WANG \& BRENNAN [13], o conhecimento do calor isostérico, em função da umidade de equilíbrio, é essencial nos estudos de secagem e armazenamento de produtos agrícolas, servindo para estimar as necessidades energéticas do processo de secagem, além de fornecer dados sobre o estado da água no produto.

Um método largamente usado para se calcular o calor isostérico de sorção, seja pelo processo de adsorção ou pelo de dessorção, é dado pela equação de Clausius-Clayperon [6], como mostrado a seguir:

$\frac{\partial \ln \left(a_{w}\right)}{\partial T}=\frac{q_{s t}}{R T^{2}}$

em que,

$\mathrm{a}_{\mathrm{w}:}$ atividade de água, decimal;

$\mathrm{T}$ : temperatura absoluta, $\mathrm{K}$;

$\mathrm{q}_{\text {st: }}$ calor isostérico líquido de sorção, $\mathrm{kJ} \mathrm{kg}^{-1}$; e

$\mathrm{R}$ : constante universal dos gases, $8,314 \mathrm{~kJ} \mathrm{kmol}^{-1} \mathrm{~K}^{-1}$, sendo para o vapor d'água $0,4619 \mathrm{~kJ} \mathrm{~kg}^{-1} \mathrm{~K}^{-1}$.

Integrando a Equação 1 e assumindo que o calor isostérico líquido de sorção é independente da temperatura, podemos encontrar o calor isostérico líquido de sorção, para cada teor de água de equilíbrio, conforme a equação a seguir [13]:

$\ln \left(\mathrm{a}_{\mathrm{w}}\right)=-\left(\frac{q_{\mathrm{st}}}{\mathrm{R}}\right) \cdot \frac{1}{\mathrm{~T}}+\mathrm{C}$

em que,

c: coeficiente do modelo.

Os valores de atividade de água, temperatura e teor de água de equilíbrio são obtidos a partir das isotermas de dessorção dos produtos em estudo. O calor isostérico integral de sorção é obtido adicionando-se aos valores de calor isostérico líquido de sorção, o valor do calor latente de vaporização da água livre de acordo com a seguinte expressão:

$Q_{s t}=q_{s t}+L=a \cdot \exp \left(-b \cdot U_{e}^{*}\right)+L$

em que,

$\mathrm{Q}_{\text {st: }}$ calor isostérico integral de sorção, $\mathrm{kJ} \mathrm{kg}^{-1}$;

L: calor latente de vaporização da água livre, $\mathrm{kJ} \mathrm{kg}^{-1}$;

$\mathrm{U}_{\mathrm{e}}{ }^{*}$ : teor de água de equilíbrio, \%b.s.; e

a, b: coeficientes do modelo.

Diante da importância do conhecimento da higroscopicidade dos produtos agrícolas e dos requerimentos de energia necessários ao processo de secagem, o objetivo no presente trabalho foi determinar as isotermas de sorção dos grãos de feijão para diversas condições de temperatura e umidade relativa do ar e ajustar diferentes modelos matemáticos aos dados experimentais, selecionando aquele que melhor representa o fenômeno, bem como obter os valores do calor isostérico de dessorção para o produto.

\section{2 - MATERIAL E MÉTODOS}

O presente trabalho foi realizado no Laboratório de Propriedades Físicas e Qualidade de Produtos Agrícolas pertencente ao Centro Nacional de Treinamento em Armazenagem (CENTREINAR), localizado na Universidade Federal de Viçosa.

Para obtenção do teor de água de equilíbrio higroscópico dos grãos de feijão, foi utilizado o método dinâmico-gravimétrico, em que um fluxo de ar, a uma dada temperatura e umidade relativa, passa pela massa de grãos. Foram utilizados grãos de feijão do grupo vermelho, colhidos manualmente, com teor de água de aproximadamente $92 \%$ (b.s.).

A secagem do produto em camada delgada foi realizada para diferentes condições controladas de temperatura (25, 35,45 e $55^{\circ} \mathrm{C}$ ) e umidade relativa do ar de secagem (entre 0,2 a 0,75 ) até que o produto atingisse o teor de água de equilíbrio com a condição do ar especificada.

As condições ambientais citadas acima, para realização dos testes, foram fornecidas por meio de uma unidade condicionadora de atmosfera, de fabricação da empresa Aminco, modelo Aminco-Aire 150/300 CFM. Foram colocadas, no interior do equipamento, bandejas removíveis com fundo telado para permitir a passagem do ar através da amostra, contendo cada uma, 50 g de produto. O fluxo de ar foi monitorado com o auxílio de um anemômetro de lâminas rotativas e mantido em torno de $10 \mathrm{~m}^{3} \mathrm{~min}^{-1} \mathrm{~m}^{-2}$. A temperatura e a umidade relativa do ar foram monitoradas por meio de um psicrômetro instalado próximo às bandejas, contendo as amostras.

Durante o processo de secagem, as bandejas com o produto foram pesadas periodicamente e o equilíbrio higroscópio foi atingido quando a variação da massa dos recipientes permaneceu aproximadamente invariável durante três pesagens consecutivas. Os teores de água do produto foram determinados pelo método gravimétrico em estufa a $105 \pm 1{ }^{\circ} \mathrm{C}$, até massa constante, em três repetições.

Aos dados experimentais da umidade de equilíbrio foram ajustados os modelos matemáticos freqüentemente utilizados para representação da higroscopicidade de produtos agrícolas, cujas equações estão apresentadas na Tabela 1 .

Para o ajuste dos modelos matemáticos, foi realizada análise de regressão não linear, pelo método Gauss Newton, utilizando-se o programa computacional STATISTICA 5.0®. Os modelos foram selecionados considerando a significância dos coeficientes de regressão pelo teste t, adotando nível de $1 \%$ de probabilidade, a magnitude do coeficiente de determinação $\left(\mathrm{R}^{2}\right)$, a magnitude do erro médio relativo $(\mathrm{P})$ e do erro 
TABELA 1 - Modelos matemáticos utilizados para predizer o fenômeno de higroscopicidade de produtos agrícolas.

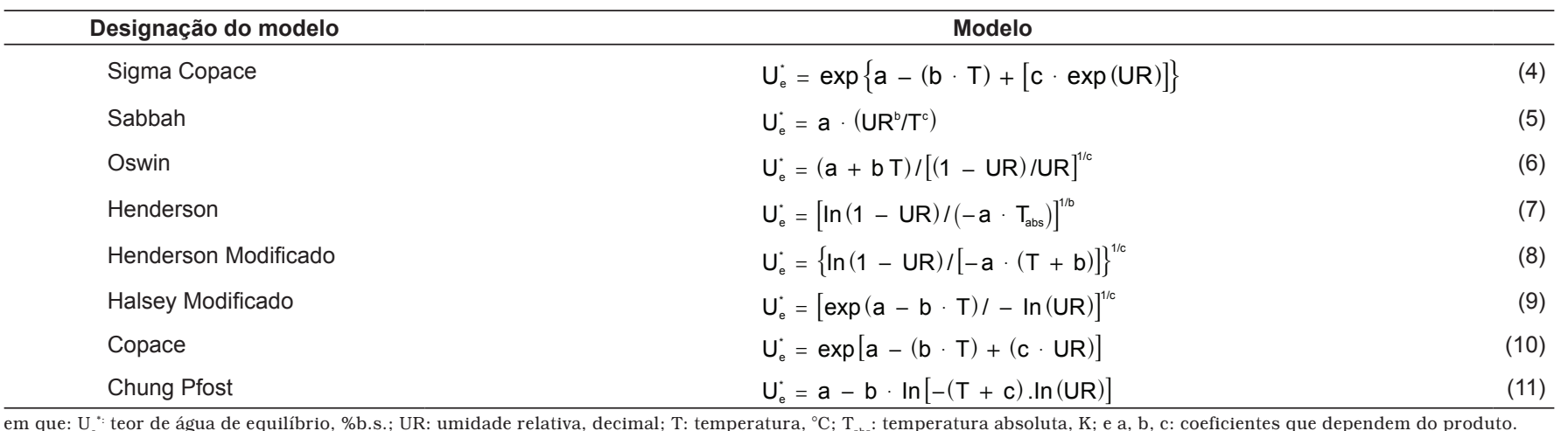

médio estimado (SE) e a verificação do comportamento da distribuição dos resíduos. Considerou-se o valor do erro médio relativo inferior a $10 \%$ como um dos critérios para seleção dos modelos, de acordo com Mohapatra e Rao [8]. O erro médio relativo e o erro médio estimado, para cada um dos modelos, foram calculados conforme as seguintes equações:

$P=\frac{100}{n} \sum_{i=1}^{n}\left(\frac{|Y-\hat{Y}|}{Y}\right)$

$S E=\sqrt{\frac{\sum_{i=1}^{n}(Y-\hat{Y})^{2}}{G L R}}$

em que:

Y: valor observado experimentalmente;

$\hat{\mathrm{Y}}$ : valor calculado pelo modelo;

n: número de observações experimentais; e

GLR: graus de liberdade do modelo (número de observações menos o número de parâmetros do modelo).

Os valores do calor isostérico líquido de dessorção $\left(\mathrm{q}_{\mathrm{st}}\right)$, para cada teor de água de equilíbrio, foram obtidos por meio da Equação 2. Os valores de atividade de água, temperatura e teor de água de equilíbrio foram obtidos a partir das isotermas de dessorção dos grãos de feijão, utilizando o modelo de melhor ajuste aos dados experimentais.

Para o cálculo dos valores do calor isostérico integral de dessorção $\left(\mathrm{Q}_{\mathrm{st}}\right)$, foi utilizada a Equação 3. O calor latente de vaporização da água livre (L), em kJ kg-1, necessário ao cálculo de $Q_{\text {st }}$, foi obtido utilizando-se a temperatura média (T) na faixa em estudo, em ${ }^{\circ} \mathrm{C}$, por meio da seguinte equação:

$L=2502,2-2,39 . T$

\section{3 - RESULTADOS E DISCUSSÃO}

Na Tabela 2, estão apresentados os parâmetros dos modelos de equilíbrio higroscópico para o feijão, obtidos por dessorção, para diferentes condições de temperatura e umidade relativa do ar.
Analisando os resultados apresentados na Tabela 2, observa-se que os modelos matemáticos utilizados para descrever a higroscopicidade do feijão apresentaram signi-

TABELA 2 - Parâmetros dos modelos de equilíbrio higroscópico para o feijão, com seus respectivos coeficientes de determinação $\left(R^{2}\right)$, erros médios estimado ( $\left.S E\right)$ e relativo $(P)$, e tendência de distribuição dos resíduos.

\begin{tabular}{|c|c|c|c|c|c|}
\hline Modelos & Parâmetros* & $\begin{array}{l}R^{2} \\
(\%)\end{array}$ & $\begin{array}{c}\mathrm{SE} \\
\text { (decimal) }\end{array}$ & $\begin{array}{l}P \\
(\%)\end{array}$ & $\begin{array}{c}\text { Distribuição } \\
\text { dos } \\
\text { Resíduos }\end{array}$ \\
\hline Sigma & $a=1,6693$ & 98,15 & 0,626 & 3,97 & Aleatória \\
\hline \multirow[t]{2}{*}{ Copace } & $b=0,0166$ & & & & \\
\hline & $c=0,8849$ & & & & \\
\hline \multirow[t]{3}{*}{ Sabbah } & $a=206,5358$ & 96,34 & 0,880 & 5,69 & Tendenciosa \\
\hline & $b=0,7640$ & & & & \\
\hline & $c=0,6264$ & & & & \\
\hline \multirow[t]{3}{*}{ Oswin } & $a=19,9325$ & 97,53 & 0,723 & 5,08 & Aleatória \\
\hline & $b=-0,1961$ & & & & \\
\hline & $c=2,7627$ & & & & \\
\hline \multirow[t]{2}{*}{ Henderson } & $a=0,0001$ & 84,74 & 1,766 & 11,72 & Tendenciosa \\
\hline & $b=1,4023$ & & & & \\
\hline \multirow{3}{*}{$\begin{array}{l}\text { Henderson } \\
\text { Modificado }\end{array}$} & $a=0,0003$ & 96,59 & 0,849 & 6,07 & Tendenciosa \\
\hline & $b=-4,2253$ & & & & \\
\hline & $c=1,7271$ & & & & \\
\hline \multirow{3}{*}{$\begin{array}{l}\text { Halsey } \\
\text { Modificado }\end{array}$} & $a=6,1927$ & 98,17 & 0,623 & 3,90 & Aleatória \\
\hline & $b=0,0348$ & & & & \\
\hline & $c=2,0973$ & & & & \\
\hline \multirow[t]{3}{*}{ Copace } & $a=2,3788$ & 97,90 & 0,666 & 4,35 & Aleatória \\
\hline & $b=0,0167$ & & & & \\
\hline & $c=1,5352$ & & & & \\
\hline \multirow{3}{*}{$\begin{array}{l}\text { Chung } \\
\text { Pfost }\end{array}$} & $a=31,3788$ & 96,65 & 1,039 & 8,18 & Tendenciosa \\
\hline & $b=6,1930$ & & & & \\
\hline & $c=-7,6554$ & & & & \\
\hline
\end{tabular}

ficância dos seus parâmetros de regressão ao nível de 5\% de probabilidade pelo teste t. Além disso, os modelos exibiram elevados valores do coeficiente de determinação (superior a 96\%), exceto o modelo de Henderson. De acordo com o critério para seleção de modelos proposto por MOHAPATRA \& RAO [8], estes modelos não se ajustaram satisfatoriamente aos dados experimentais (erro médio relativo superior a $10 \%)$, indicando serem inadequados para a descrição do fenômeno estudado. 
Ainda na Tabela 2, verifica-se que, dentre a série testada, apenas os modelos de Sigma-Copace, Oswin, Halsey Modificado e Copace apresentaram distribuição aleatória dos resíduos, indicando ajuste mais adequado aos dados experimentais. Dentre estes, o modelo de Halsey Modificado exibiu maior coeficiente de determinação e menores valores de erros médios relativo e estimado, sendo recomendado para predição do equilíbrio higroscópico dos grãos de feijão. Resultados semelhantes foram observados por CHEN [3] e CHEN \& MOREY [4] que recomendam o modelo de Halsey Modificado para estimar o equilíbrio higroscópico dos grãos de amendoim. Entretanto, AJIBOLA et al. [1] observaram que o modelo Henderson Modificado representou adequadamente o equilíbrio higroscópio do feijão caupi, e Oliveira, CAVALCANTI MATA \& DUARTE [11] recomendaram os modelos Henderson modificado e Oswin Modificado para a descrição da higroscopicidade do feijão macassar verde.

Na Figura 1, estão apresentados os valores experimentais da umidade de equilíbrio do feijão, obtidos por dessorção, bem como suas isotermas calculadas pelo modelo de Halsey Modificado.

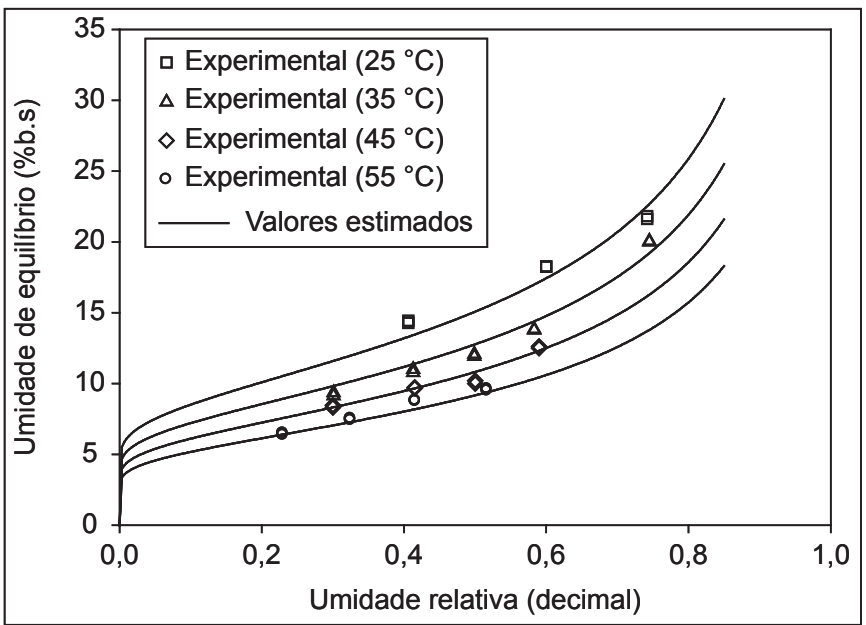

FIGURA 1 - Isotermas de dessorção de água para os grãos de feijão, estimadas pelo modelo de Halsey Modificado, para diferentes condições de temperatura e umidade relativa do ar.

Observa-se na Figura 1 que para uma umidade relativa constante os valores de umidade de equilíbrio diminuíram com o aumento da temperatura, seguindo a mesma tendência da maioria dos produtos agrícolas.

Diante da representação satisfatória do equilíbrio higroscópico dos grãos de feijão, o modelo de Halsey Modificado foi utilizado para o cálculo dos valores de atividade de água, necessários para a determinação dos valores do calor isostérico líquido de dessorção.

Na Tabela 3, estão apresentadas as umidades relativas de equilíbrio (atividade de água), obtidas por meio do modelo de Halsey Modificado, para os grãos de feijão com teores de água variando de 10,10 a 21,71 (\%b.s.) e para a faixa de temperatura de 25 a $55{ }^{\circ} \mathrm{C}$.
TABELA 3 - Valores de atividade de água (decimal) estimados pelo modelo de Halsey Modificado em função da temperatura e da umidade de equilíbrio.

\begin{tabular}{cccccccc}
\hline $\mathbf{U}_{\mathbf{e}}$ (\%b.s.) & \multicolumn{7}{c}{ Temperatura $\left({ }^{\circ} \mathbf{C}\right)$} \\
& $\mathbf{2 5}$ & $\mathbf{3 0}$ & $\mathbf{3 5}$ & $\mathbf{4 0}$ & $\mathbf{4 5}$ & $\mathbf{5 0}$ & $\mathbf{5 5}$ \\
\hline 10,10 & 0,20 & 0,26 & 0,32 & 0,39 & 0,45 & 0,51 & 0,57 \\
10,88 & 0,25 & 0,32 & 0,38 & 0,44 & 0,50 & 0,56 & 0,62 \\
11,99 & 0,33 & 0,39 & 0,45 & 0,51 & 0,57 & 0,63 & 0,67 \\
12,56 & 0,36 & 0,43 & 0,49 & 0,55 & 0,60 & 0,65 & 0,70 \\
13,80 & 0,43 & 0,50 & 0,56 & 0,61 & 0,66 & 0,71 & 0,75 \\
14,35 & 0,46 & 0,52 & 0,58 & 0,63 & 0,68 & 0,72 & 0,76 \\
18,26 & 0,63 & 0,68 & 0,72 & 0,76 & 0,79 & 0,82 & 0,85 \\
20,02 & 0,68 & 0,73 & 0,76 & 0,80 & 0,83 & 0,85 & 0,87 \\
21,71 & 0,72 & 0,76 & 0,80 & 0,83 & 0,85 & 0,87 & 0,89 \\
\hline
\end{tabular}

Na Figura 2, são apresentadas as curvas do logaritmo neperiano da atividade de água (decimal), para específicos valores de umidade de equilíbrio (\%b.s.), em função de valores do inverso da temperatura absoluta $(\mathrm{K})$ para os grãos de feijão.

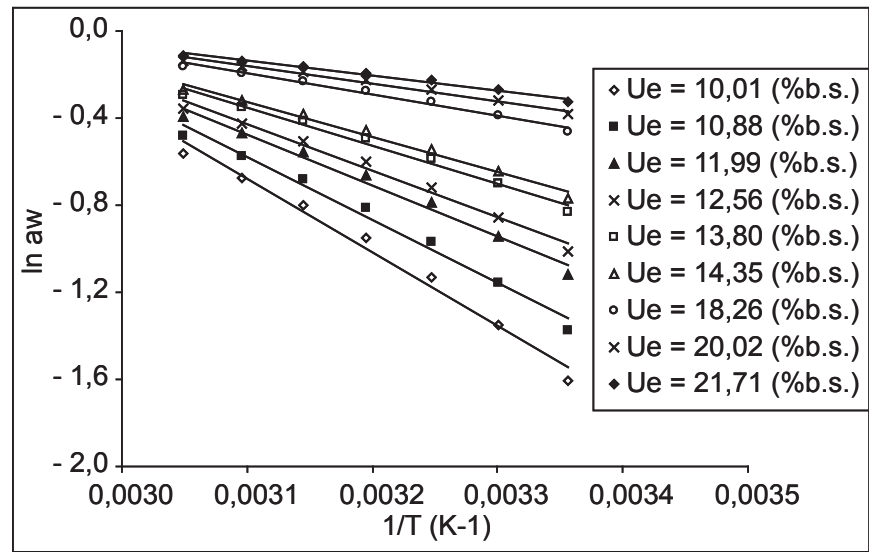

FIGURA 2 - Curvas do logaritmo neperiano da atividade de água (decimal), para específicos valores de umidade de equilíbrio (\%b.s.), em função de valores de $1 / T\left(K^{-1}\right)$ para os grãos de feijão.

Os valores do calor isostérico líquido de dessorção $\left(\mathrm{q}_{\mathrm{st}}\right)$, em kJ kg-1, para os grãos de feijão, em função do teor de água de equilíbrio (\%b.s.), foram calculados de acordo com a Equação 2, representados, para cada situação, pelas inclinações das retas ilustradas na Figura 2. Para o calor isostérico integral de dessorção $\left(Q_{\mathrm{st}}\right)$, em $\mathrm{kJ} \mathrm{kg}^{-1}$, foram adicionados, aos valores de $\mathrm{q}_{\mathrm{st}}$, o valor do calor latente de vaporização da água livre (L), que representa a mínima quantidade de energia necessária para evaporar a água em estado livre, calculado para temperatura média de $40{ }^{\circ} \mathrm{C}$, resultando no valor de 2406,60 $\mathrm{kJ} \mathrm{kg}^{-1}$.

Na Figura 3, são apresentados os valores do calor isostérico integral de dessorção $\left(Q_{\mathrm{st}}\right)$, em kJ kg-1, em função do teor de água de equilíbrio (\%b.s.), estimados de acordo com a Equação 3. 


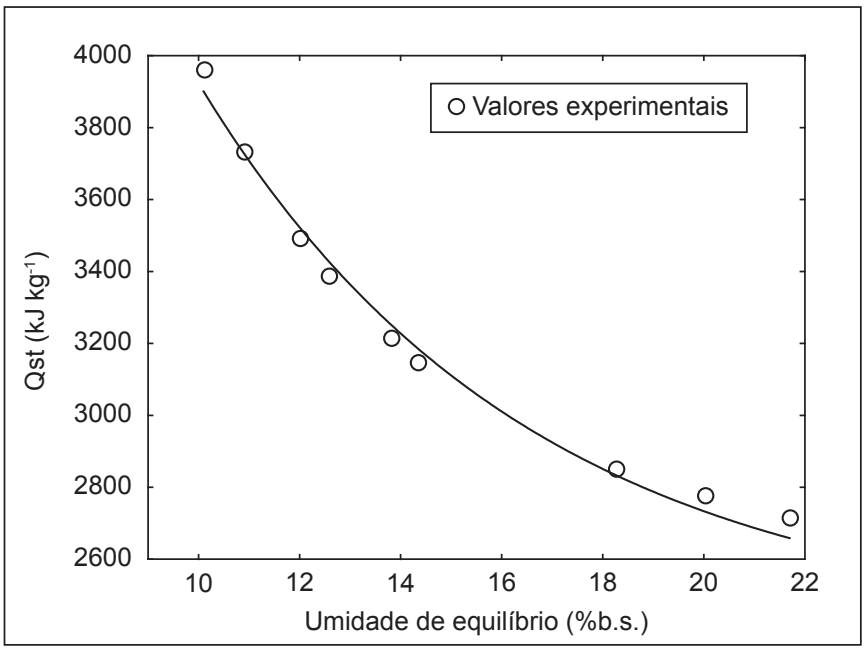

FIGURA 3 - Calor isostérico integral de dessorção para os grãos de feijão em função do teor de água de equilíbrio.

De acordo com a Figura 3, observa-se que, com a redução do teor de água, ocorre aumento da energia necessária para a remoção de água do produto, representada pelos valores do calor isostérico integral de dessorção $\left(Q_{\mathrm{st}}\right)$, conforme observado para diversos produtos agrícolas [6, $9,12,7]$.

Os valores de calor isostérico integral de dessorção, para os grãos de feijão na faixa de umidade de 10,10 a 21,71 (\%b.s.), variaram de 3961 a $2718 \mathrm{~kJ} \mathrm{~kg}^{-1}$. Estes valores estão coerentes com os encontrados por AYRANCI \& DUMAN [2], que, trabalhando com feijão caupi na faixa de umidade de 4 a 16 (\%b.s.), obtiveram valores de calor isostérico integral de dessorção variando de 3361 a $2472 \mathrm{~kJ} \mathrm{~kg}^{-1}$, aproximadamente.

As possíveis diferenças encontradas entre os valores observados de calor isostérico integral de dessorção para os diferentes produtos, podem ser justificadas, além dos fatores inerentes aos próprios produtos, segundo FERREIRA \& PENA [5], por possíveis erros na obtenção dos valores de atividade de água, para cada teor de água de equilíbrio, uma vez que os valores foram obtidos a partir de modelo matemático ajustado. Apesar destas discrepâncias, ZHANG et al. [14], estudando isotermas de sorção de umidade de alguns vegetais nas temperaturas de 30 a $60^{\circ} \mathrm{C}$, concluíram que o calor isostérico de dessorção pode ser utilizado para estimar a energia requerida no processo de desidratação de produtos agrícolas.

Na Tabela 4, está apresentado o modelo matemático para os valores experimentais do calor isostérico integral de dessorção $\left(Q_{\mathrm{st}}\right)$, em kJ kg $\mathrm{kg}^{-1}$, em função do teor de água de equilíbrio (\%b.s.). Observa-se que a equação utilizada se mostrou satisfatória na descrição do fenômeno, apresentando elevada significância dos seus parâmetros e do coeficiente de determinação $\left(\mathrm{R}^{2}\right)$.
TABELA 4 - Modelo de regressão ajustado aos valores experimentais do calor isostérico integral de dessorção $\left(Q_{\mathrm{st}}\right)$, em kJ kg-1, em função do teor de água de equilíbrio (\%b.s.), com seus respectivos coeficiente de determinação $\left(\mathrm{R}^{2}\right)$ e níveis de significância.

\begin{tabular}{cl}
\hline Modelo & $\mathbf{R}^{2}(\%)$ \\
\hline$Q_{\mathrm{st}}=7039,35^{* *} \cdot \exp \left(-0,153^{* *} \cdot U_{\mathrm{e}}^{\star}\right)+2406,6$ & $99,02^{++}$ \\
\hline
\end{tabular}

${ }^{* *}$ Significativo a $1 \%$ de probabilidade, pelo teste $\mathrm{t}$; $\mathrm{e}^{++}$Significativo a $1 \%$ de probabilidade, pelo teste $\mathrm{F}$.

\section{4 - CONCLUSÕES}

Com base nos resultados obtidos, conclui-se que:

- o teor de água de equilíbrio higroscópico do feijão é diretamente proporcional à umidade relativa do ar e decresce com o aumento de temperatura, para uma mesma umidade relativa, seguindo a mesma tendência da maioria dos produtos agrícolas já estudados;

- baseando-se em parâmetros estatísticos, o modelo de Halsey Modificado é o que melhor representa a higroscopicidade do produto estudado, quando comparado aos modelos tradicionalmente utilizados para descrição deste fenômeno; e

- com a redução do teor de água, ocorre aumento da energia necessária para a remoção de água do produto, sendo que os valores do calor isostérico integral de dessorção, para os grãos de feijão na faixa de umidade de 10,10 a 21,71 (\%b.s.), variam de 3961 a $2718 \mathrm{~kJ} \mathrm{~kg}^{-1}$.

\section{5 - REFERÊNCIAS BIBLIOGRÁFICAS}

[1] AJIBOlA, O. O.; AVIARA N. A.; AJETUMOBI, O. E. Sorption equilibrium and thermodynamic properties of cowpea (Vigna unguiculata). Journal of Food Engineering, v. 58, p. 317-324. 2003.

[2] AYRANCI, E.; DUMAN, O. Moisture sorption isotherms of cowpea (Vigna unguiculata L. Walp) and its protein isotate at 10,20 and $30^{\circ} \mathrm{C}$. Journal of Food Engineering, v. 70, p. 83-91, 2005.

[3] CHEN, C. A rapid method to determine the sorption isotherms of peanuts. Journal Agricultural Engineering Research, New York, v. 75, p. 401-408, 2000.

[4] CHEN, C.; MOREY, R. V. Comparison of four EMC/ERII equacions. Transaction of the ASAE, St. Joseph, v. 32, n. 3, p. 983-990, 1989.

[5] FERREIRA, C. D.; PENA, R. S. Comportamento higroscópico da farinha de pupunha (Bactris gasipaes). Ciência e Tecnologia de Alimentos, Campinas, v. 23, n. 2, p. 251-255, 2003.

[6] IGLESIAS, H.; CHIRIFE, J. Prediction of the effect of temperature on water sorption isotherms of food material. Journal of Food Technology, Oxford, v. 11, p. 109-116, 1976.

[7] JAYENDRA KUMAR, A.; SINGH, R. R. B.; PATIL, G. R.; PATEL, A. A. Effect of temperature on moisture desorption isotherms of kheer. Food Science and Technology, v. 38, p. 303-310, 2005.

[8] MOHAPATRA, D.; RAO, P. S. A thin layer drying model of parboiled wheat. Journal of Food Engineering. p. 513-518, 2005. 
[9] MUlet, A.; GARCIA-REVERTER, J.; SANJUÁN, R.; BON, J. Sorption isosteric heat determination by thermal analysis and sorption isotherms. Journal of Food Science, v. 64, n. 1, p. 64-68, 1999.

[10] MULET, A.; GARCYA-PASCUAL, P.; SANJUÁN, N.; GARCÝA-REVERTER, J. Equilibrium isotherms and isosteric heats of morel (Morchella esculenta). Journal of Food Engineering, London, v. 53, p. 75-81, 2002.

[ 11 ] OLIVEIRA, J. R.; CAVALCANTI MATA, M. E. R.; DUARTE, M. E. M. Isotermas de dessorção de grãos de feijão macassar verde (Vigna unguiculata (L.) Walpers), variedade sempre-verde. Revista Brasileira de Produtos Agroindustriais, Campina Grande, v. 6, n. 1, p. 61-70, 2004.
[12] TOLABA, M. P.; PELTZER, M.; ENRIQUEZ, N.; POLLIO, M.L. Grain sorption equilibria of quinoa grains. Journal of Food Engineering, v. 61, p. 365-371, 2004.

[13] WANG, N.; BRENNAN, J. G. Moisture sorption isotherm characteristics of potato at four temperatures. Journal of Food Engineering, v. 14, p. 269-287, 1991.

[14] ZHANG, X.; LIU, X.; GU, D.; ZHOU, W.; WANG, R.; MARINOS-KOURIS, D. Desorption isotherms of some vegetables. Journal of Science and Food Agriculture, v. 70, p. 303-306, 1996.

\section{6 - AGRADECIMENTOS}

Os autores agradecem à CAPES, ao CNPq e à FAPEMIG pelo apoio financeiro indispensável na execução do presente trabalho. 